\section{Is no SNUs good news?}

\section{Lawrence M. Krauss}

ANY hopes that the long-standing 'solar neutrino' problem might go away once new neutrino detectors came on line appear to have faded with the first results from the Soviet-American Gallium Experiment (SAGE), reported in Physical Review Letters last month ${ }^{1}$. The authors report a deficit in the number of neutrinos coming from the Sun, compared with the number expected to be produced by the main solar nuclearenergy-producing reactions. This result supports previous evidence that something is wrong with our understanding of either solar or neutrino physics, and further points towards the latter.

The neutrinos that are being sought are emitted directly from the solar core, where they are produced in nuclear reactions which fuel the Sun. Because the light we see is emitted from the solar surface, it is only by detecting neutrinos that we can probe directly the physical processes associated with solar energy production, and thus solar evolution. The SAGE experiment is located in a deep underground laboratory at the Baksan Neutrino Observatory in the north Caucasus. It involves detecting the production of about one atom of ${ }^{91} \mathrm{Ge}$ per day in a detector containing 30 tons of liquid ${ }^{71} \mathrm{Ga}$, produced by interactions of electron neutrinos $\left(v_{\mathrm{c}}\right)$ originating in fusion reactions which convert hydrogen to helium.

To date, there have been two other solar neutrino experiments - a radiochemical experiment involving the production of ${ }^{37} \mathrm{Ar}$ from ${ }^{37} \mathrm{Cl}$ in an underground tank at the Homestake gold mine in the United States ${ }^{2}$, which has been taking data for over 20 years; and a real-time detector of neutrino events in a large underground water tank at the Kamioka facility in $\mathrm{Japan}^{3}$, which has been reporting results for three years. These have both consistently reported neutrino event rates substantially below those predicted by standard solar models, leading to the solar neutrino problem. The solution could require dramatic new physics associated with either astrophysical processes deep in the solar interior, or the properties of neutrinos themselves. In either case, the consequences could be profound.

The crucial feature that differentiates the ${ }^{71} \mathrm{Ga}$ experiment from the earlier solar neutrino experiments is the fact that the latter are primarily sensitive to only the highest-energy component of the solar neutrino flux, one associated with a rather minor side reaction $\left({ }^{8} \mathrm{~B} \rightarrow\right.$ $\left.{ }^{8} \mathrm{Be}+\mathrm{e}^{-}+v_{\mathrm{e}}\right)$ in the nuclear chain that causes four hydrogen nuclei to fuse and has done just that, T. R. mical Society (Atlanta, more massive? make the nucleus of a helium atom. On the other hand, the threshold $(0.2332$ $\mathrm{MeV})$ for the reaction $v_{\mathrm{c}}+{ }^{71} \mathrm{Ga} \rightarrow{ }^{71} \mathrm{Ge}$ $+\mathrm{e}^{-}$is extremely low, allowing the ${ }^{71} \mathrm{Ga}$ experiment to be primarily sensitive to the dominant component of the neutrino flux from the Sun. This component is associated with the first step of the fusion reaction chain $\left({ }^{1} \mathrm{H}+{ }^{1} \mathrm{H} \rightarrow{ }^{2} \mathrm{H}+\right.$ $\left.\mathrm{e}^{+}+v_{\mathrm{c}}\right)$, and so is directly linked to the solar luminosity, a well measured quantity. Hence, any suppression of this neutrino signal would give unambiguous evidence that the solar neutrino problem originates in the fundamental physics of neutrinos. But were the observed signal to agree with that predicted, it might suggest that the solar neutrino problem is restricted to the high-energy boron nuclear reaction network, or perhaps that the other detectors have been giving spurious results.

For these reasons, the SAGE results have been eagerly awaited. The data have been a long time in the making. The basic chemistry associated with the ${ }^{71} \mathrm{Ga}$ experiment was first explored 14 years ago ${ }^{4}$. SAGE uses four Teflon-lined chemical reactors, each holding about 7 tons of liquid gallium. For each measurement a small amount of natural germanium carrier is added to each reactor; after 3-4 weeks this carrier and any ${ }^{71} \mathrm{Ge}$ atoms produced are chemically extracted as $\mathrm{GeCl}_{4}$, after the addition of hydrochloric acid and hydrogen peroxide, and this is synthesized and purified into germane gas $\left(\mathrm{GeH}_{4}\right)$. The overall extraction efficiency of germanium by this procedure is about 80 per cent. The germane gas is mixed with xenon, and then put into low-background gas proportional counters. These in turn are placed in shields, where they are sensitive to the $\mathrm{X}$-rays and electrons produced when ${ }^{71} \mathrm{Ge}$ atoms radioactively decay, and monitored for 2-3 months.

\title{
Black hole is M87's bright spot
}

WHEN they reported in 1978 the possible discovery of a black hole in the elliptical galaxy M87, W. L. W. Sargent et al. suggested that the projected Space Telescope would provide the extra spatial resolution needed to confirm their claim. Now, 14 years later, the much maligned Hubble Space Telescope Lauer, S. M. Faber and colleagues claimed at the recent meeting of the American Astrono-

13-17 January 1992). The evidence comes from the brightening of the galactic light near the nucleus. This same brightening was seen in the earlier observations of Sargent et al., who attributed it to the increasing density of stars in that direction. Comparing the stellar distribution with that in a normal galaxy, those authors concluded that the anomalously concentrated packing of stars, and their velocities (revealed spectroscopically), could be accounted for only if there was a central concentration, probably a black hole, comprising 5 billion $\left(10^{9}\right)$ solar masses in the nucleus. The new high-resolution image shown here, taken with the Hubble's Wide Field Camera, confirms that picture, and the astronomers conclude that the black hole is equivalent to 2.6 billion solar masses. Some of the reasons why M87 was chosen in the search for a massive black hole are also apparent in the figure. The galaxy is the archetypal radio or active galaxy. At its centre is a brilliant source of radio waves, shown here aiso to be the source of intense (non-stellar) optical emission. Also it ejects one of the most dramatic astrophysical jets, stretching 5,000 light years to the right on this picture. This, and possibly its counterpart, illuminate two giant radio lobes that extend beyond the visible extent of the galaxy. All this activity is thought to result from a disk of dense plasma in the galactic core, heated as it spirals onto the central black hole. The Hubble scientists promise further spectroscopic studies to strengthen their conclusion. Another reason for looking at M87 is that it is relatively close, so affording us a good view. Will Hubble now be turning its cameras towards NGC6240, which is even closer and may contain, it was suggested last year, a black hole ten times 